\title{
AVALIAÇÃO DA DISTRIBUIÇÃO ESPACIAL DA TUBERCULOSE NA REGIÃO DO PONTAL DO PARANAPANEMA
}

Anne Beatriz Bortoluci ${ }^{1}$, Jamile Silveira Tomiazzi ${ }^{1}$, Rosana Amaral Carrasco ${ }^{2}$, Ana Paula Marques Ramos ${ }^{1}$, Marcus Vinicius Pimenta Rodrigues ${ }^{1}$

Universidade do Oeste Paulista - UNOESTE, ${ }^{1}$ Mestrado em Meio Ambiente e Desenvolvimento Regional, Presidente Prudente, SP. ${ }^{2}$ Curso de Engenharia Produção, Presidente Prudente, SP. E-mail: anne@unoeste.br

\section{RESUMO}

A tuberculose é uma doença infecciosa crônica, com ampla distribuição mundial, que há muito tempo afeta a humanidade e representa, ainda hoje, grave problema de saúde pública. Trata-se de uma doença negligenciada, endêmica em diversos países e responsável por cerca de 1,3 milhões de óbitos anualmente. Atualmente, o Brasil ocupa o 160 lugar na classificação de países campeões em casos de tuberculose. 0 mapeamento de doenças é uma ferramenta fundamental, pois, a partir do conhecimento da distribuição espacial das doenças, podem-se definir ações preventivas ou mesmo corretivas para o combate das mesmas. No estado de São Paulo, há áreas muito carentes de mapeamento cartográfico da tuberculose, uma das quais é a região do Pontal do Paranapanema. $O$ objetivo deste trabalho foi realizar a distribuição espacial da tuberculose na região do Pontal do Paranapanema no período de 2007 a 2014 . O método utilizado foi a associação da taxa média de incidência da tuberculose neste período. As unidades de análises foram os 32 municípios da região do Pontal do Paranapanema. Os dados foram levantados da Sala de Apoio a Gestão Estratégica (SAGE). Quanto aos dados cartográficos, utilizou-se os disponíveis pelo Instituto Brasileiro de Geografia e Estatística (IBGE). Dentre os anos estudados, observaram-se taxas de incidência entre 7,9 e 299,49 para cada 100.000 habitantes. Há evidências da relação da tuberculose com alguns fatores, dentre eles as unidades prisionais. Conclui-se que a distribuição da tuberculose nos 32 municípios da área de estudo não é homogênea. Diante disso, cabe ressaltar a necessidade em realizarem-se estudos que correlacionem a distribuição espacial com os aspectos epidemiológicos da tuberculose.

Palavras-chave: tuberculose, cartografia, epidemiologia, Pontal do Paranapanema.

\section{SPATIAL DISTRIBUTION EVALUATION OF TUBERCULOSIS IN THE PONTAL DO PARANAPANEMA AREA.}

\begin{abstract}
Tuberculosis is a chronic infectious disease with worldwide distribution, which affects the humanity for long time and is, today, a serious public health problem. This is a neglected disease, endemic in several countries and accounts for about 1.3 million deaths annually. Brazil is 16th place in the ranking of countries in cases of tuberculosis currently. The disease mapping is a key tool because from the knowledge of the spatial distribution of diseases can be defined preventive or corrective actions to combat them. In São Paulo, there are very poor areas of cartographic mapping of tuberculosis, one of which is the Pontal do Paranapanema region. The aim of this work is to perform the spatial distribution of tuberculosis in the Pontal do Paranapanema region from 2007 to 2014 . The method used was the combination of the average rate of tuberculosis incidence in this period. The analysis units were the 32 municipalities in the Pontal do Paranapanema region. The data were collected from the Support Strategic Management Room (SAGE). As for the map data, we used available by the Brazilian Institute of Geography and Statistics (IBGE). Among the years studied, they observed incidence rates between 7.9 and 299.49 per 100,000 inhabitants. There is evidence of the relation of tuberculosis with some factors, including the prisons. We conclude that the distribution of tuberculosis in the 32 municipalities of the study area is not homogeneous. Therefore, it points out the need to be hold studies that correlate the spatial distribution with the epidemiology of tuberculosis.
\end{abstract}

Keywords: tuberculosis, cartography, epidemiology, Pontal do Paranapanema. 


\section{INTRODUÇÃO}

A tuberculose é uma doença infecciosa crônica, com ampla distribuição mundial, e que há muito tempo afeta a humanidade, representando, ainda hoje, um grave problema de saúde pública. Diferente do que se imaginou nas décadas de 1960 e 1970, de que, com a conquista de uma potente quimioterapia, a doença tenderia a um efetivo controle, a tuberculose recrudesceu em todo mundo (LEITE, 2009). Esta doença possui alta incidência e mortalidade, apesar de ser passível de prevenção e tratamento de forma eficiente, situação resultante do abandono do tratamento, do surgimento de cepas resistentes, da epidemia do HIV (Vírus da Imunodeficiência Humana) e das condições socioeconômicas desfavoráveis. Um cenário que dificulta o controle da doença e prejudica seu tratamento, aumentando, assim, a susceptibilidade e a vulnerabilidade do indivíduo (PAIXÃO et al., 2007).

O Plano Nacional de Controle da Tuberculose, lançado pelo Ministro da Saúde, em 1999, define a tuberculose como prioridade entre as políticas governamentais de saúde, estabelece diretrizes para as ações e fixa metas para o alcance de seus objetivos (DA SILVA, 2004). Esta é uma doença negligenciada e endêmica em diversos países, sendo responsável por cerca de 1,3 milhões de óbitos anualmente (World Health Organization, 2013). Segundo o Ministério da Saúde (2009), estima-se que um terço da população mundial esteja infectada pelo Mycobacterium tuberculosis - bacilo aeróbico que apresenta alta infectividade e baixa patogenicidade - estando, portanto, sob risco de desenvolver a doença e que, aproximadamente, $95 \%$ dos casos de adoecimento ocorram nos países em desenvolvimento.

Embora a tuberculose esteja presente em todas as regiões do mundo, aquelas que detêm a maior incidência são Ásia (58\%) e África (27\%), com predominância do número de casos na Índia, China e África do Sul. Em 2012, a incidência global estimada para a tuberculose foi de 122 casos por 100.000 habitantes, totalizando cerca de 12 milhões de casos no mundo. Neste mesmo ano, o Brasil encontrava-se entre os 22 países com maior índice de doença, com uma taxa de incidência de 37,3 casos a cada 100.000 habitantes, o que corresponde a 72.309 casos. Além disso, casos na forma pulmonar foram de 30,7 a cada 100.000 habitantes, em um total de 59.518 ocorrências (World Health Organization, 2013).

Dentre as regiões brasileiras, as que têm maior taxa de incidência são a Norte, a Sudeste e o Nordeste que alcançaram, neste mesmo ano de 2012, as taxas de 38,4; 32,6 e 30,8 casos a cada 100.000 habitantes, respectivamente. Tais taxas foram responsáveis por classificar o Brasil no $13^{\circ}$ lugar em números absolutos de casos de tuberculose e a totalizar 35\% dos casos notificados no continente sul americano (World Health Organization, 2013). No Estado de São Paulo, a taxa de incidência no ano de 2012 foi de 37,3 casos a cada 100.000 habitantes, totalizando 15.615 casos. Em 2014 foram registrados 67.966 casos de tuberculose no Brasil, com coeficiente de incidência de 33,5 casos a cada 100.000 habitantes. Atualmente, o Brasil encontra-se no 16 o lugar na classificação de países campeões em casos dessa doença. No Estado de São Paulo, a taxa de incidência corresponde a 37,2 a cada 100.000 habitantes, o que equivale a 16.370 casos (BRASIL - MINISTÉRIO DA SAÚDE, 2016).

A associação da Medicina com a Cartografia é bastante antiga, bem como o ato de explorar o potencial das informações veiculadas pelos mapas em um processo de busca do entendimento do dinamismo espacial das doenças. O mapeamento das doenças é fundamental quando se considera a necessidade de vigilância diante de uma epidemia, pois o conhecimento do padrão geográfico das doenças pode fornecer informações sobre a etiologia e a fisiopatologia de determinados eventos mórbidos (NETO; COMETTI, 2007). O cenário atual da aplicação do Geoprocessamento nos estudos da saúde no Brasil é extremamente favorável e pode ser sumarizado segundo quatro eixos de desenvolvimento: a disponibilização de bases de dados, os programas disponíveis, o desenvolvimento tecnológico e a capacitação de pessoal (FAUSTO et al., 2010). O termo Geoprocessamento denota a disciplina do conhecimento que utiliza técnicas matemáticas e ferramentas computacionais para o tratamento da informação espacial, sendo tais ferramentas computacionais os Sistemas de Informação Geográfica (SIG) (CÂMARA; DAVIS, 2004).

O Estado de São Paulo está dividido em 24 áreas administrativas, de acordo com o Decreto $\mathrm{n}^{\circ}$ 40.083 de 15/05/1995, denominadas Direções Regionais de Saúde (DRS). Cada área geográfica foi enumerada englobando uma quantidade de municípios recebendo o nome do município sede (MENCARONI, 2003). O Pontal do Paranapanema é uma região de 18844,60 km² que compreende 32 municípios do Estado de São Paulo e está localizada no extremo oeste do estado, região sudeste do Brasil (Figura 1). A população total do Pontal é de 583.703 habitantes, sendo $89,74 \%$ desse total concentrada em áreas urbanas, segundo o Censo do ano de 2010 do Instituto Brasileiro de Geografia e Estatística (IBGE). 
No estado de São Paulo, há três áreas muito carentes do mapeamento da distribuição espacial da tuberculose, uma das quais é a região do Pontal do Paranapanema. Assim, o presente trabalho tem como objetivo realizar a distribuição espacial da tuberculose na região do Pontal do Paranapanema, entre os anos de 2007 a 2014.

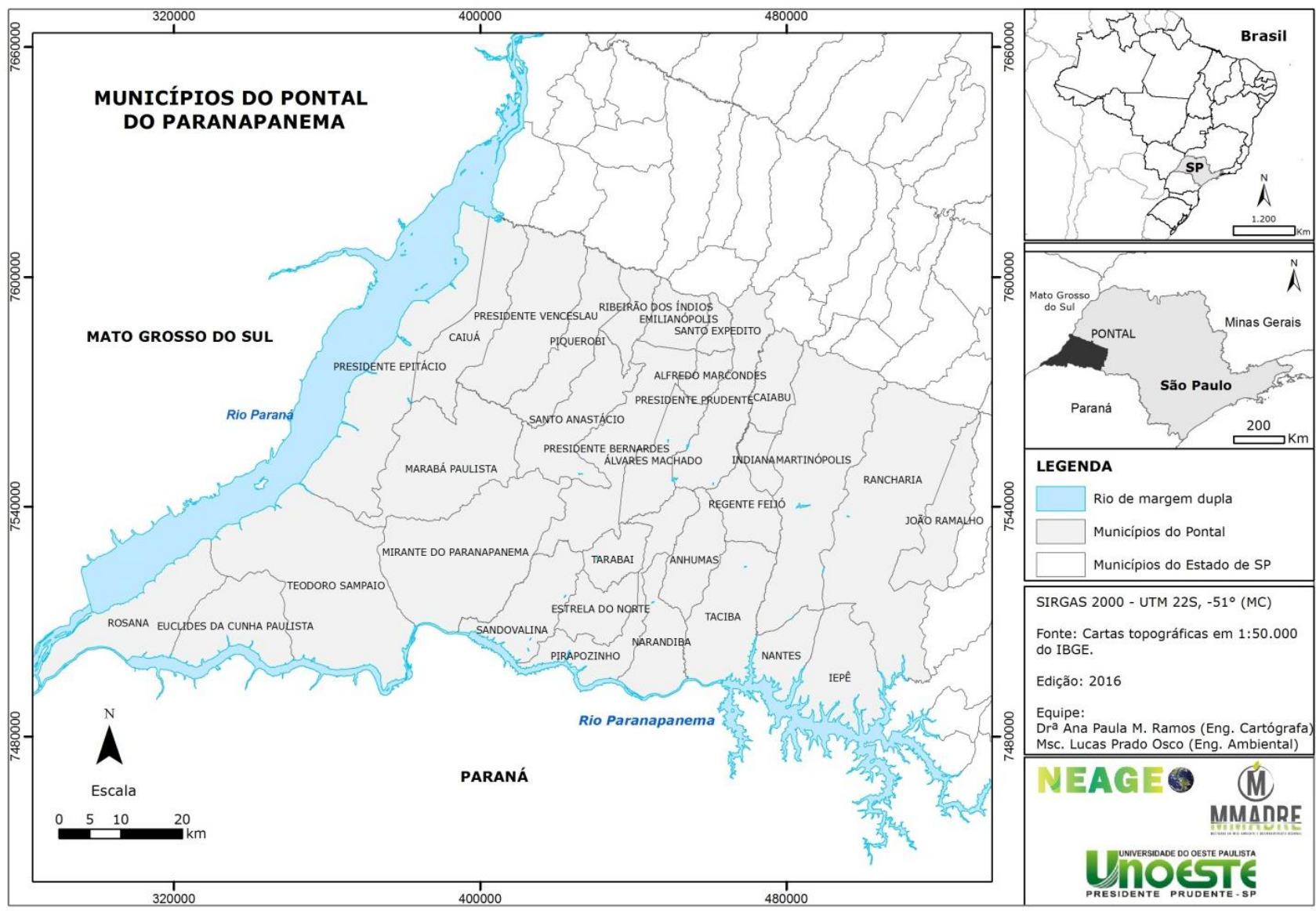

Figura 1. Localização do Pontal do Paranapanema no Estado de São Paulo.

\section{METODOLOGIA}

Os materiais utilizados na realização desta pesquisa consistiram em: software de Sistema de Informação Geográfica, base de dados vetoriais dos municípios e dados alfanuméricos da taxa de incidência da tuberculose. $O$ método constituiu-se de três etapas: coleta dos dados de tuberculose para a área de estudo; organização dos dados e produção do mapa da taxa média de incidência da tuberculose na região do Pontal do Paranapanema entre os anos de 2007 a 2014.

Os dados referentes à taxa de incidência foram obtidos por meio da base de dados da Sala de Apoio a Gestão Estratégica (SAGE) (Figura 2). Foram selecionados apenas os casos novos de tuberculose, no período de 2007 a 2014 . A SAGE estima a taxa de incidência dividindo o número de casos novos de tuberculose pela população do município no ano de estudo, multiplicado pelo fator de 100.000 . Para o caso de estudo considerou-se a média da taxa de incidência de tuberculose dos anos de 2007 a 2014. 


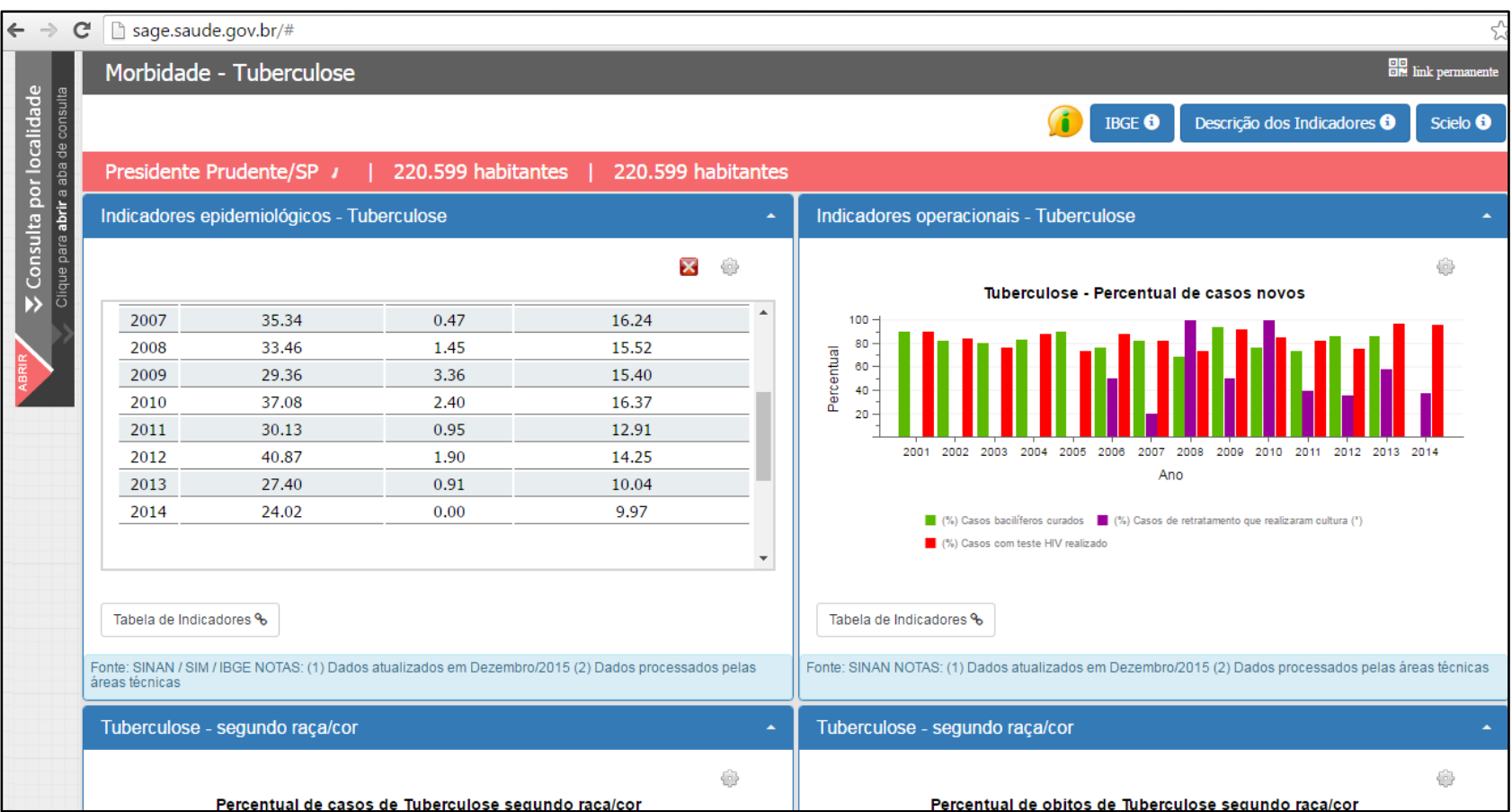

Figura 2. Coleta de dados na Sala de Apoio à Gestão Estratégica (SAGE).

Fonte: http://www.saude.gov.br/sage

A organização dos dados foi realizada no Microsoft Excel por municípios, ou seja, para cada um dos 32 municípios do Pontal organizou-se a taxa média de incidência de tuberculose (Figura 3). Para a produção do mapa da taxa média de incidência da tuberculose entre os anos de 2007 a 2014, utilizou-se uma base cartográfica dos munícipios do Pontal do Paranapanema, em formato shapefile, disponibilizada no site do IBGE conforme mostra a Figura 4. A partir dos dados tabulares, e aplicando-se funções de Geoprocessamento, em software de SIG, podem-se unir tais dados à base vetorial de municípios e, posteriormente, elaborar os mapas temáticos de interesse.

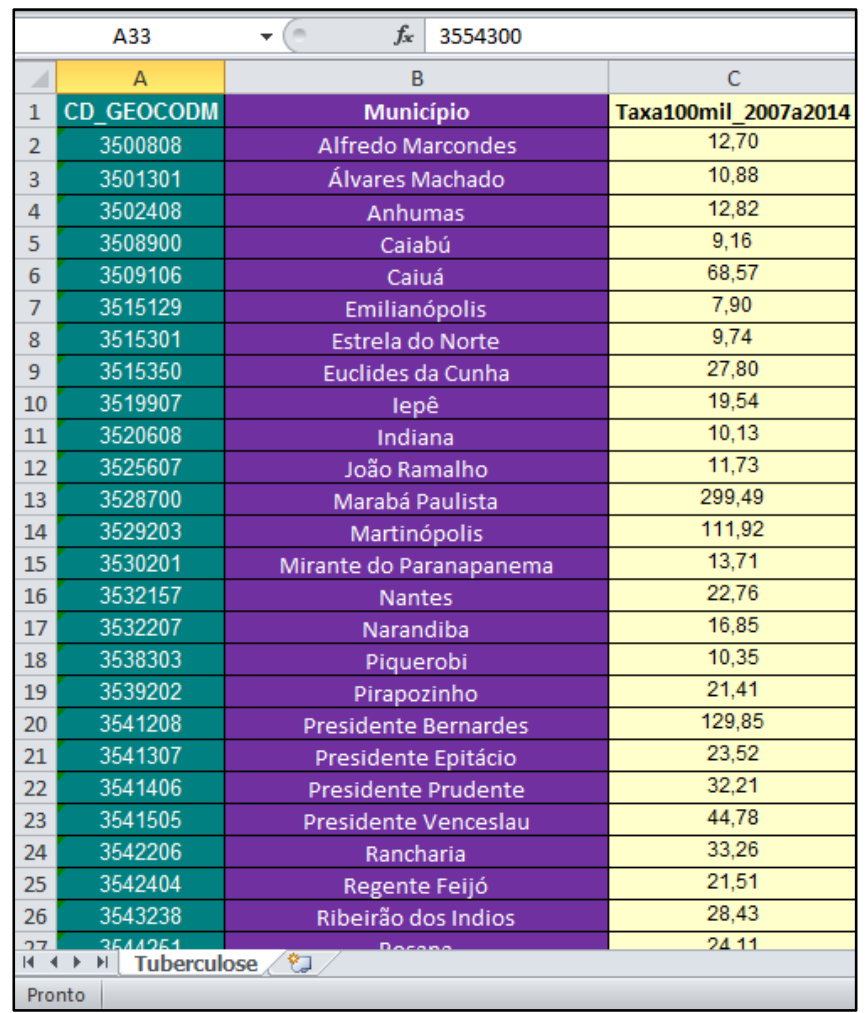

Figura 3. Tabulação dos dados em planilha do Microsoft Excel. 


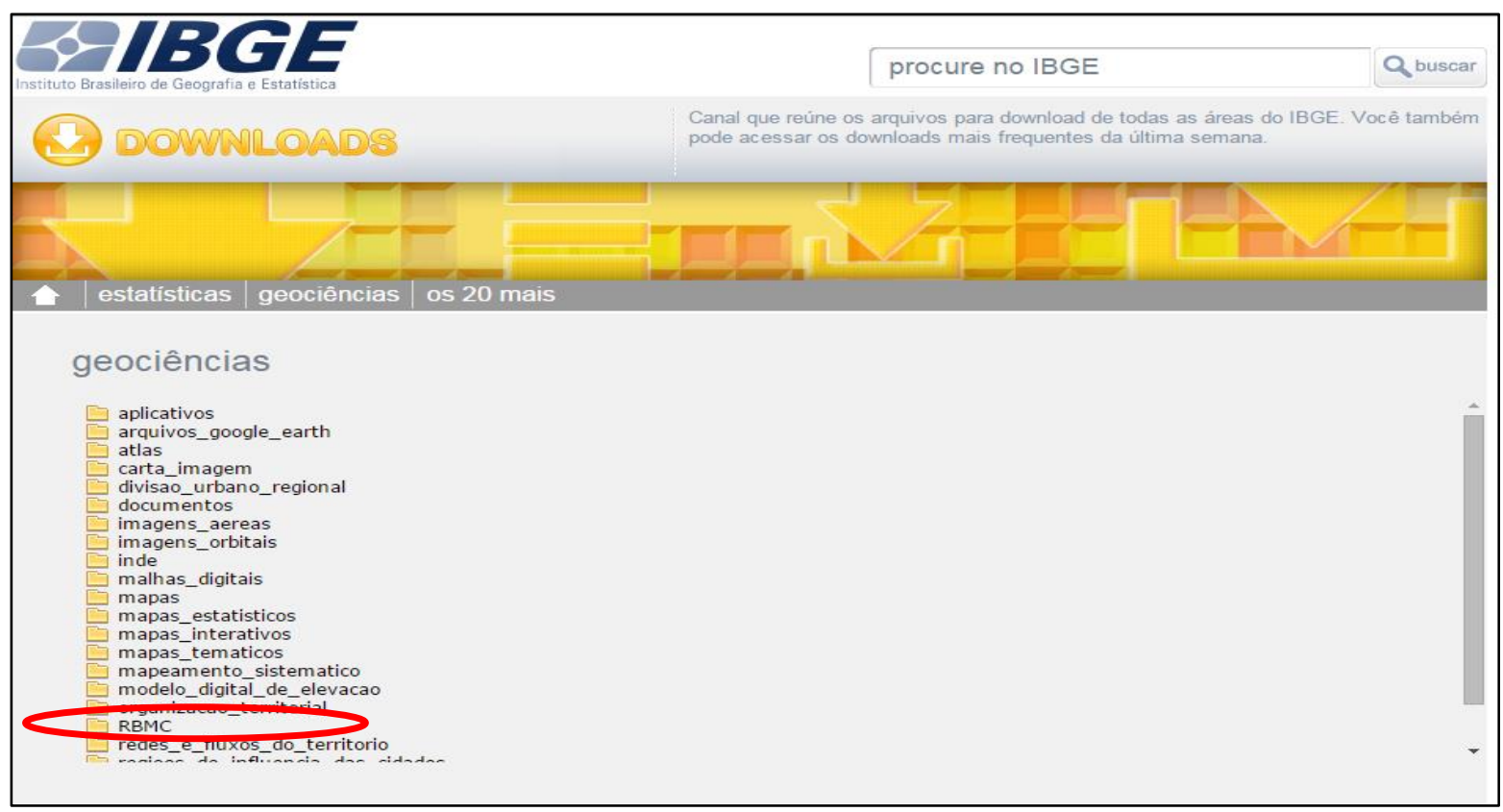

Figura 4. Base de dados do IBGE.

Fonte: http://www.ibge.gov.br

\section{RESULTADOS E DISCUSSÃO}

A Figura 5 apresenta o mapa temático da distribuição espacial da taxa média de incidência da tuberculose entre os anos de 2007 a 2014, na região do Pontal do Paranapanema. Trata-se de um mapa temático quantitativo, elaborado pelo método coroplético, utilizando classificação manual com cinco classes, segundo a abordagem de Dent; Torguson; Hodler, (2009).

Entre os anos estudados, foram observadas taxas de incidência entre 7,9 e 299,49 as quais correspondem aos municípios de Emilianópolis e de Marabá Paulista, respectivamente. Apesar da baixa incidência analisada na região do Pontal, observa-se uma distribuição espacial heterogênea quando comparado os municípios. Dentre os municípios avaliados, citam-se os quais apresentaram maiores taxas média de incidência da tuberculose, quais sejam: Caiuá $(68,57)$, Martinópolis $(111,92)$, Presidente Bernardes $(129,85)$ e Marabá Paulista $(299,49)$.

Segundo Xavier et al (2007), estudos evidenciam a relação da tuberculose com desigualdades sociais, estilo de vida, especificamente, em população mais pobres e aglomeração de pessoas, tais como: comunidades, assentamentos e acampamentos rurais, bem como com a localização de unidades prisionais.

Cabe ressaltar que a região do Pontal do Paranapanema é uma região que abriga uma grande quantidade de acampamentos e assentamentos rurais apresentando o maior número de assentamentos do Brasil, com mais de 110 projetos já instalados e, aproximadamente, 6,2 mil famílias assentadas, segundo informações do banco de dados da Luta pela Terra (BARONE; MELAZZO; SILVA, 2011).

Adicionalmente, a região abrange o maior número de unidades prisionais do país (Secretaria da Administração Penitenciária, 2016). Quando comparado com as regiões mais industrializadas do Estado, a região do Oeste Paulista apresenta um fraco índice de urbanização e apresenta o menor Produto Interno Bruto (PIB), resultando em uma região de grande assimetria socioeconômica. Esta situação é agravada pelo fato do crescimento populacional das cidades não ter, como contrapartida, o desenvolvimento equânime de políticas públicas, principalmente nas áreas de Educação e de Saúde.

Nos quatro municípios que apresentaram as maiores taxas média da incidência de tuberculose, observou-se um aspecto em comum entre os mesmos: a instalação de unidades prisionais. A Tabela 1 monstra a ocupação das unidades prisionais na região do Pontal do Paranapanema. Ressalta-se a superlotação nas unidades prisionais dos municípios de Presidente Prudente, Caiuá, Marabá Paulista e Martinópolis, onde três desses municípios apresentaram altas taxas média de incidência da tuberculose. 


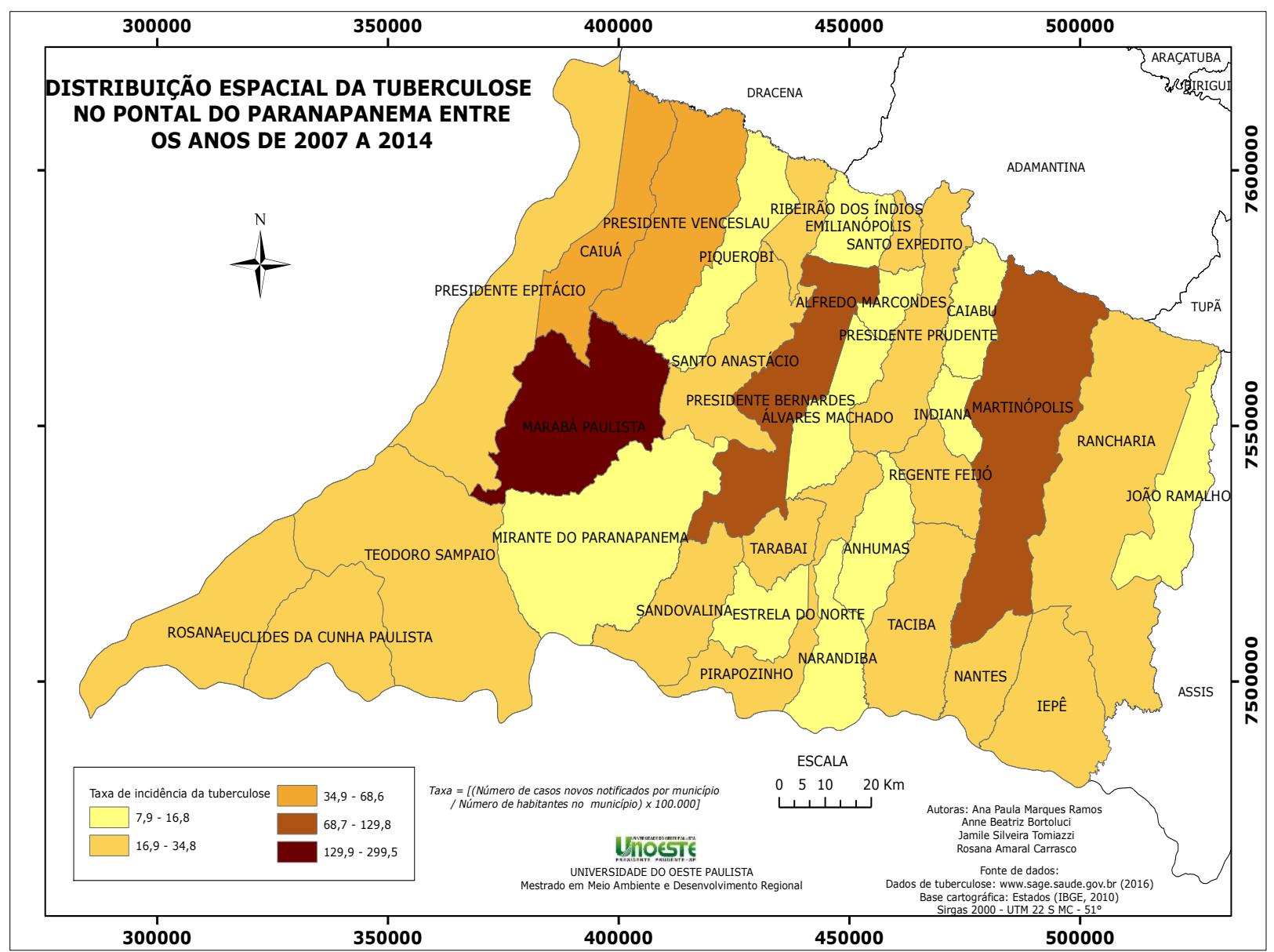

Figura 5. Distribuição espacial da tuberculose no Pontal do Paranapanema entre os anos de 2007 a 2014.

Tabela 1. Análise da população carcerária nas Unidades Prisionais dos municípios do Pontal do Paranapanema.

\begin{tabular}{c|c|c|c}
\hline $\begin{array}{c}\text { Município / População } \\
\text { (CENSO, 2010) }\end{array}$ & $\begin{array}{c}\text { Capacidade máxima } \\
\text { da população } \\
\text { carcerária }\end{array}$ & $\begin{array}{c}\text { Total de } \\
\text { sentenciados } \\
\text { em 2015 }\end{array}$ & $\begin{array}{c}\text { Porcentagem de } \\
\text { encarcerados em função do } \\
\text { número de habitantes do } \\
\text { município }\end{array}$ \\
\hline $\begin{array}{c}\text { Presidente Prudente / } \\
207.610 \text { habitantes }\end{array}$ & 943 sentenciados & 1517 & $7,3 \%$ \\
\hline $\begin{array}{c}\text { Presidente Bernardes / } \\
13.570 \text { habitantes }\end{array}$ & 204 sentenciados & 181 & $13,3 \%$ \\
\hline $\begin{array}{c}\text { Caiuá / } \\
5.039 \text { habitantes }\end{array}$ & 844 sentenciados & 1210 & $24,0 \%$ \\
\hline $\begin{array}{c}\text { Presidente Venceslau / } \\
37.910 \text { habitantes }\end{array}$ & $\begin{array}{c}\text { Penitenciaria I: } 781 \\
\text { sentenciados }\end{array}$ & 830 & $2,2 \%$ \\
\hline $\begin{array}{c}\text { Presidente Venceslau / } \\
37.910 \text { habitantes }\end{array}$ & $\begin{array}{c}\text { Penitenciaria II: } 1280 \\
\text { sentenciados }\end{array}$ & 839 & $37,7 \%$ \\
\hline $\begin{array}{c}\text { Marabá Paulista / } \\
4.182 \text { habitantes }\end{array}$ & 844 sentenciados & 1578 & $7,86 \%$ \\
\hline $\begin{array}{c}\text { Martinópolis / } \\
\text { 24.219 habitantes }\end{array}$ & 872 sentenciados & 1906 & \\
\hline
\end{tabular}

Fonte: Secretaria Estadual de Administração Penitenciária 2016 e IBGE 2010

A principal razão para o alto índice de doenças contagiosas nesses nas unidades prisionais pode ser a aglomeração. Neste aspecto, o Brasil conta uma taxa de incidência de 299,7 privados de liberdade para cada 100.000 habitantes, dado este que deixa o país em $4^{\circ}$ na classificação de população carcerária (MINISTÉRIO DA JUSTIÇA, 2016). Na população em geral, cada doente não coberto com tratamento de 
tuberculose tende a infectar de 10 a 15 pessoas em um ano, e uma ou duas manifestam a doença, fazendo com que ela permaneça na população como endemia (BREDA, 1999). Somam-se a isso as condições de confinamento, já que ambientes fechados, com pouca ventilação e muita concentração de pessoas, são altamente favoráveis à disseminação da tuberculose, aumentando o risco de contágio (GLASER et al., 1993). Diante disso, não somente para os detentos nas unidades prisionais quanto para as pessoas que trabalham nesses locais, tem-se um maior risco de se contrair doenças infectocontagiosas, tais como hepatites virais, HIV/AIDS, sífilis e a tuberculose. Essa dinâmica se intensifica devido às precárias condições de vida nas unidades prisionais, juntamente com condições sanitárias adversas, baixo nível socioeconômico e o uso de substâncias ilícitas que favorecem a ocorrência de adoecimento e morte (CONINX et al., 1999). Além disso, algumas práticas, como o consumo de drogas injetáveis e o sexo sem proteção, contribuem para a transmissão da infecção pelo HIV (GLASER et al., 1993). Desse modo, uma associação da tuberculose com elevadas taxas de infecção por HIV entre os privados de liberdade pode conduzir a uma epidemia substancial de tuberculose no sistema prisional, a menos que medidas ativas de controle sejam tomadas para neutralizar a propagação da doença (NYANGULU et al., 1997).

O impacto causado pela tuberculose para a saúde pública é inegável e extremamente importante, já que suas consequências são diretas. Diante disso, são necessárias políticas públicas destinadas a atender essa demanda específica.

Dessa maneira, o mapeamento da quantificação da tuberculose por município pode auxiliar no planejamento ambiental e urbano, atuando como ferramenta para indicar a alocação de recursos e direcionar possíveis estratégias de intervenção.

\section{CONCLUSÃO}

Este trabalho realizou a distribuição espacial da taxa média de incidência da tuberculose na região do Pontal do Paranapanema, entre 2007 a 2014. A taxa de incidência da tuberculose apresentou-se elevada em alguns municípios, mostrando que essa doença ainda representa um grave problema de saúde pública.

Um dos fatores que mais contribuem para essa problemática na região do Pontal do Paranapanema pode estar relacionado com a instalação de unidades prisionais, onde quatro destas apresentaram superlotação de sentenciados. Isto acarreta em aglomerações de pessoas contribuindo para a disseminação da doença. Com isso, observa-se a necessidade da inclusão de políticas públicas destinadas a atender essa demanda específica.

Conclui-se que a distribuição espacial da tuberculose nos 32 municípios em estudo não é homogênea e recomenda-se novos estudos que correlacionem essa distribuição com aspectos epidemiológicos da tuberculose. Essa correlação é extremamente importante, pois fundamenta a definição de indicadores, a elaboração e a execução de estratégias de intervenção para o controle da doença. Conclui-se, também, que a Cartografia, por meio do Geoprocessamento, viabilizou a análise dos casos de tubérculos, a qual, até o presente momento, é ainda considerado um problema de saúde pública no Brasil e no mundo. Sugere-se a aplicação de outros tipos de análise espacial com os dados, como por exemplo, Índice Global de Moran, a fim de se verificar se a localização geográfica do município influencia na taca de tuberculose.

\section{CONFLITO DE INTERESSE}

Os autores declaram não haver qualquer potencial de conflito de interesse que possa interferir na imparcialidade deste trabalho científico.

\section{REFERÊNCIAS}

BARONE, L. A; MELAZZO, E. S; SILVA, A. A. Célula do Pontal do Paranapanema - SP. Acompanhamento e Informação para o desenvolvimento rural. Ministério do Desenvolvimento Agrário. Presidente Prudente, $\mathrm{n}$ 5, p. 1-116, 2011.

BRASIL. Manual de recomendações para o controle da tuberculose no Brasil. Ministério da Saúde. Secretaria de Vigilância em Saúde. Departamento de Vigilância Epidemiológica. 2016.

BREDA, J.G. Recomendações para o controle da tuberculose em prisões. São Paulo: 1999. 
CÂMARA, G.; DAVIS, C. Introdução. In: CÂMARA, G. et al. Introdução à Ciência da Geoinformação. INPE, Cap.1.2004.

CONINX, R. et al. First-line tuberculosis therapy and drug resistant Mycobacterium tuberculosis in prisons. The Lancet. v. 353, n. 9157, p. 969-73, 1999.

DA SILVA JR, J.B., Tuberculose: Guia de vigilância epidemiológica. Jornal Brasileiro de Pneumologia. p. 5785. v. 30 (Suppl 1), jun. 2004.

DENT, B. D.; TORGUSON, J.; HODLER, T. Cartography: Thematic Map Design. $6^{\text {th }}$ ed. McGraw-Hill, Georgia, p. 368. 2009.

FAUSTO, C.A.S. et al. Análise clínica, epidemiológica e espacial de pacientes com hanseníase em Lagoa Grande/PE, por meio de tecnologias da geoinformação. In: SIMPÓSIO BRASILEIRO DE CIÊNCIAS GEODÉSICAS E TECNOLOGIAS DA GEOINFORMAÇÃO, 3.,2010, Recife - PE, Anais eletrônicos... Recife, 2010.

GLASER, J.B. et al. Correctional health care: a public health opportunity. Ann Inter Med, n. 118, v. 2, p. 13945, 1993.

Instituto Brasileiro de Geografia e Estatística (IBGE). Censo 2010. Brasília: IBGE, 2016. Disponível em: <http://www.ibge.gov.br>. Acesso em: 15 mai. 2016.

LEITE, A.M.T., Caracterização clínica-epidemiológica dos pacientes com diagnóstico de tuberculose atendidos em um serviço de referência em Maceió, Alagoas. 2009. 52 f. Monografia (Especialização em Saúde da Família) - Instituto A Vez do Mestre, Universidade Candido Mendes, Rio de Janeiro.

MENCARONI, D.A. Análise Espacial da Endemia Hansênica no Município de Fernandópolis/SP. 2003. 126 f. Tese (Enfermagem em Saúde Pública) - Escola de Enfermagem de Ribeirão Preto, Universidade de São Paulo, Ribeirão Preto.

Ministério da Justiça. Departamento Penitenciário Nacional. Sistema Penitenciário no Brasil. Dados consolidados. Brasília, 2016.

Ministério da Saúde, Secretaria de Vigilância em Saúde, Departamento de Vigilância Epidemiológica. Guia de vigilância epidemiológica. 7. Ed. Brasília, 2009.

NETO, A.A.B.; COMETTI, R.R. Sensoriamento remoto como ferramenta auxiliar no combate à ocorrência de dengue na cidade de Vitória-ES. In: SIMPÓSIO BRASILEIRO DE SENSORIAMENTO REMOTO, 13, 2007, Florianópolis. Anais eletrônicos... Florianópolis, 2007.

NYANGULU, D.S. et al. Tuberculosis in a prison population in Malawi. The Lancet. v. 350, n. 11, p. 1284-7, 1997.

PAIXÃO, L.M.M., GONTIJO, E.D., Perfil de casos de tuberculose notificados e fatores associados ao abandono, Belo Horizonte, MG, Revista de Saúde Pública, São Paulo, v. 41, n. 2, p. 205-213, abr., 2007. https://doi.org/10.1590/S0034-89102007000200006

Sala de Apoio a Gestão Estratégica - SAGE. Ministério da Saúde. Secretaria de Vigilância em Saúde. Departamento de Vigilância Epidemiológica. Brasília: SAGE, 2016. Disponível em: <http://www.saude.gov.br/sage>. Acesso em: 15 mai. 2016.

Secretaria da Administração Penitenciária - SAP. São Paulo, 2016. Disponível em: <http://www.sap.sp.gov.br/> Acesso em: 23 jun. 2016. 
World Health Organization. Global tuberculosis report 2013. Geneva; 2013. Disponível em: <http://apps.who.int/iris/bitstream/10665/91355/1/9789241564656_eng.pdf>. Acesso em: 15 mai. 2016.

XAVIER, M. I. M.; BARRETO, M. L. Tuberculose na cidade de Salvador, Bahia, Brasil: o perfil na década de 1990. Cad. Saúde Pública, Rio de janeiro, v. 23, n. 2, p. 445-453, fev. 2007. 